\title{
Why Genomics Shouldn't Get Too Personal: In Favor of Filters
}

Gabrielle M Christenhusz, ${ }^{1}$ Koenraad Devriendt, ${ }^{2}$ Joris Vermeesch, ${ }^{2}$ Kris Dierickx ${ }^{1}$

${ }^{1}$ Centre for Biomedical Ethics and Law, Catholic University of Leuven, Belgium

${ }^{2}$ Centre for Human Genetics, Catholic University of Leuven, Belgium

Corresponding author: Gabrielle M Christenhusz,

Centre for Biomedical Ethics and Law, Kapucijnenvoer 35, 3000 Leuven, Belgium

Telephone: +32 16-332919 Fax: +32 16-33.69.52

Email: gabrielle.christenhusz@med.kuleuven.be 


\section{Why Genomics Shouldn't Get Too Personal: In Favor of Filters}

Leuven

26 January 2012

Dear Sir/Madam

Re: Invited Comment by Holly K. Tabor et al. in American Journal of Medical Genetics Part A volume 155

We read with great interest the recent "invited comment" by Holly K. Tabor et al. on the new ethical challenges raised by exome sequencing and whole genome sequencing (ES/WGS) [Tabor et al., 2011]. We are in general agreement with their position that ES/WGS fundamentally challenge assumptions valid for other genetic sequencing techniques in the areas of informed consent, data sharing and unanticipated findings. What we would like to add is the query whether it is in fact desirable to generate so many unanticipated findings.

While Tabor et al. acknowledge that ES/WGS results with potential clinical utility may not have actual utility, and that the return of ES/WGS results will be a strain on resources financial, time, and expertise - their starting premise appears to be that these are obstacles to be overcome. We wish to suggest an alternative starting premise: the number of ES/WGS results of potential clinical utility should be limited as far as possible; concerns about actual utility and the strain to resources then become secondary.

We base this premise on several arguments:

1. Good scientific conduct - the primary objective of scientific research is to complete a scientific project. While, as Tabor et al. state, the aim of some ES/WGS research 
projects is "to identify potentially deleterious alleles in all protein-coding genes [or] the entire genome," even these projects need specific aims. A potentially overwhelming number of unanticipated findings threatens the feasibility of ES/WGS research projects to achieve their specified aims [Kohane et al., 2006].

2. Information overload in clinical contexts - information overload is not just a threat to the future feasibility of ES/WGS research. The increasingly intricate, ambiguous and complex unanticipated findings stemming from ES/WGS applications in clinical care will also demand increases in time and staff expertise. The consequence is that not all findings can be analyzed and communicated. [Ali-Khan et al., 2009]

3. The principle of distributive justice - the identification, verification and communication of ES/WGS findings can take resources away from primary research and primary healthcare. This is especially an issue when there are already great strains on primary healthcare in developing countries [Dickert and Wendler, 2009], and when research and healthcare are largely publically rather than privately funded.

Furthermore, in developed/high-income countries, smoking, sedentary behavior and excessive food and alcohol consumption cause more morbidity and premature mortality than genetically determined conditions; it would appear to be a fairer distribution of resources to devote more funding to research on behavioral change than ES/WGS research. [Evans et al., 2011]

4. Who pays? - While there are high expectations that ES/WGS will soon be readily affordable for both consumers and the ordinary healthcare system, there are also predictions that "the $\$ 1000$ genome may create a million dollar headache" in terms of interpretation and follow-up costs [Sharp, 2011]. Whose responsibility is it to pay this? In addition, once ES/WGS become so affordable and widespread, the danger of overmedicalization arises, in which everyone has genetic anomalies and complaints but no one necessarily has clinical conditions that can be treated or prevented. Whose responsibility is it to pay for the consequences of over-medicalization? 
Given these challenges to research, clinical care and entire healthcare systems posed by an over-abundance of ES/WGS results, we argue that the number of potential ES/WGS results be limited by another approach: the imposition of a filter system. This filter system would make a distinction between the raw sequencing data and information that has been analyzed. Information in the raw sequencing data is only relevant after it has been analyzed. This targeted interrogation of the raw sequencing data is conducted in relation to a certain health risk or a certain indication. As a result, only results of clear clinical benefit will be sought by the relevant researchers or clinicians, and only these results will have to be verified and communicated. The filter system should be applied in consultation with a clinician who is in regular contact with the subject in question such as the subject's family doctor. This involvement is to ensure the link between results of potential clinical utility and those of actual clinical utility, because the family doctor will be familiar with the subject's clinical history. The implementation of a filter system will force ES/WGS to a great extent into existing genetic paradigms. While some may argue that this will restrict the potential of new genetic technologies at a research level too much, [Evans, 2011] we are convinced that the problems caused by an indiscriminate generation of ES/WGS findings will otherwise be too great. The development and implementation of a filter system will answer the four arguments offered above. In limiting the number of unanticipated findings to those that are clinically relevant and actionable, good scientific conduct will be safeguarded, increases in time and staff expertise will be limited, the distributive justice argument is answered, and the costs of interpretation and follow-up plus the potential danger of over-medicalization are restrained.

Gabrielle M Christenhusz BSc MA, Koenraad Devriendt MD PhD, Joris Vermeesch PhD, Kris Dierickx PhD 


\section{References}

Ali-Khan S, Daar A, Shuman C, Ray PN, Scherer SW. 2009. Whole genome scanning: resolving clinical diagnosis and management amidst complex data. Pediatr Res. 66(4): 357-363.

Dickert N. and Wendler D. 2009. Ancillary care obligations of medical researchers. JAMA 302(4): 424-428.

Evans JP. 2011. Looking ahead, looking behind. Introduction. Genet Med 13(3): 177-178.

Evans JP, Meslin EM, Marteau TM, Caulfield T. 2011. Genomics. Deflating the genomic bubble. Science 331(6019): 861-862.

Kohane I, Masys D, Altman RB. 2006. The incidentalome: a threat to genomic medicine. JAMA 296(2): 212-215.

Sharp, R. R. 2011. Downsizing genomic medicine: approaching the ethical complexity of whole-genome sequencing by starting small. Genet Med 13(3): 191-194.

Tabor HK, Berkman BE, Hull SC, Bamshad MJ. 2011. Genomics really gets personal: How exome and whole genome sequencing challenge the ethical framework of human genetics research. Am J Med Gen Part A 155(12): 2916-2924. 